\title{
Frequency Modulation of Body Waves to Improve Performance of Limbless Robots
}

\author{
Baxi Chong*, Tianyu Wang ${ }^{\dagger}$, Jennifer M. Rieser*, Abdul Kaba ${ }^{\ddagger}$, Howie Choset $^{\dagger}$ and Daniel I. Goldman* \\ ${ }^{*}$ Georgia Institute of Technology \\ ${ }^{\dagger}$ Carnegie Mellon University \\ ${ }^{\ddagger}$ Morehouse College
}

\begin{abstract}
Sidewinder rattlesnakes generate movement through coordinated lateral and vertical traveling waves of body curvature. Previous biological and robotic studies have demonstrated that proper control and coordination of these two waves enables robust and versatile locomotion in complex environments. However, the propagation of the vertical wave, which sets the bodyenvironment contact state, can affect static stability and lead to undesirable locomotion behaviors, especially for movement at low speeds. Here, we propose to stabilize gaits by modulations of the spatial frequency of the vertical wave, which can be used to tune the number of distinct body-environment contact patches (while maintaining a constant overall contact area). These modulations act to stabilize configurations that were previously statically unstable and therefore, by eliminating dynamic effects such as undesired turning, broaden the range of movements and behaviors accessible to limbless locomotors at a variety of speeds. Specifically, our approach identifies, for a given lateral wave, the spatial frequency of the vertical wave that statically stabilizes the locomotor and then uses geometric mechanics tools to identify the coordination (i.e., the phase shift) between the vertical and lateral waves that produces a desired motion. We demonstrate the effectiveness of our technique in robot experiments.
\end{abstract}

\section{INTRODUCTION}

Many biological limbless locomotors successfully maneuver over complex terrain, partially because of their capability to regulate the body contact pattern with the environment [1, 4, 8, 9, 12, 18]. Proper coordination of dynamics in the lateral plane via body contact regulation can lead to a variety of effective locomotion behaviors, such as sidewinding [12], sinus-lift slithering [8], rapid turning (for example, reversal turning [4] and frequency turning [1]), and gradual turning (differential turning [1]).

Despite the potential benefits to maneuverability, lifting certain body segments can often result in the loss of static stability, as the locomotor's center of mass can leave the support polygon [14]. When locomotors are operating at high speed, the loss of static stability can be compensated by dynamic stability such that the duration of the unstable configuration is too short to affect the robot's overall dynamics [13]. However, at low speed, the loss of static stability can lead to unexpected body contact and change the ground reaction force distribution, which often leads to undesirable deviations from the expected locomotion performance.

Marvi et al. [12] demonstrated that on granular media, modulating the amplitude of motions in the vertical plane can increase the amount of the body in contact with the

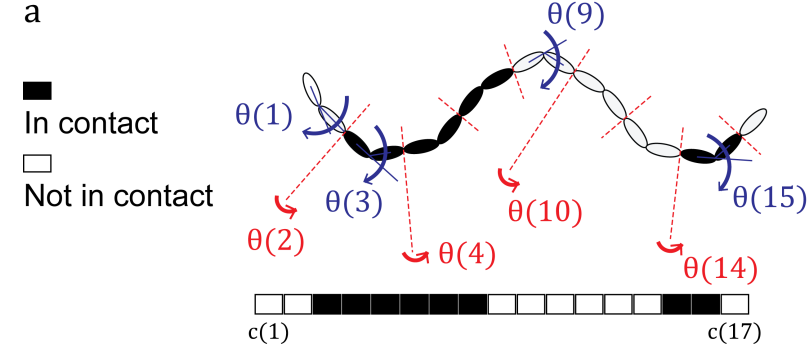

b. 1

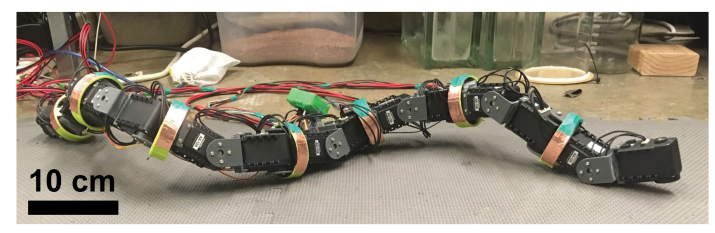

b.2

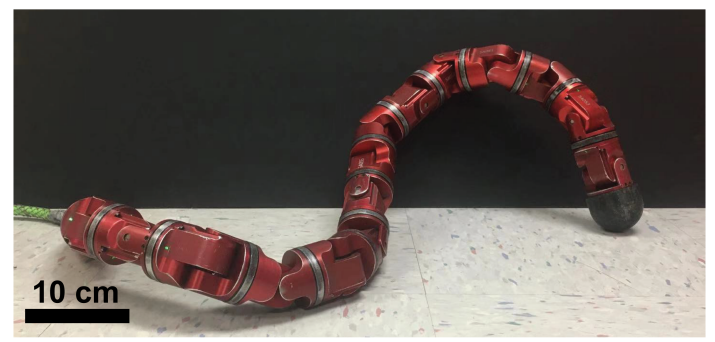

Fig. 1. Theoretical Model and Experimental Robots (a) The theoretical model for the sidewinder robots. The filled black ovals indicate the ground contact phase while the white ovals indicate a no ground contact phase. The contact state is labelled in black $(c(i))$. The joint angle in blue indicates vertical joints and the joint angle in red indicates lateral joints. (b.1) The Dynamixel robot [2] used to test the effect of static stability on the sidewinding gaits. (b.2) The serial elastic actuated (SEA) robot used to test the effectiveness of our stabilization approach.

environment and therefore stabilize the gaits. However, on flat hard ground, amplitude modulation does not directly change the contact length and therefore cannot stabilize gaits on nondeformable flat substrates. We propose an approach to stabilize the statically unstable gaits by modulating the distribution of the body contact pattern (frequency modulation). However, the changes in the body contact distribution can change the distribution of the ground reaction forces and therefore produce motions in undesired directions [1]. To address the change in the contact distribution, proper coordination of motions in the 


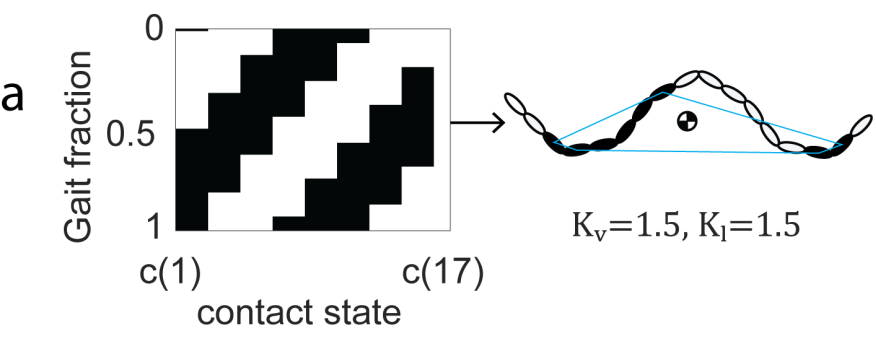

b.1
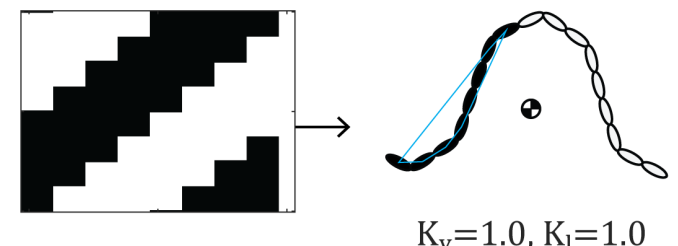

b.2
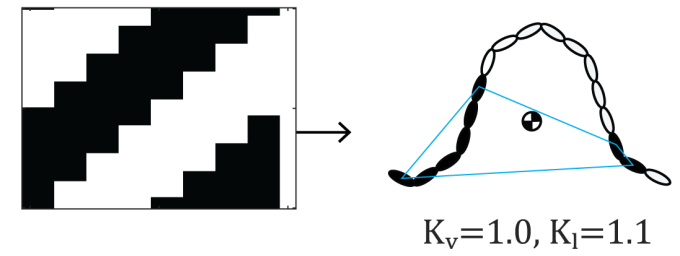

C
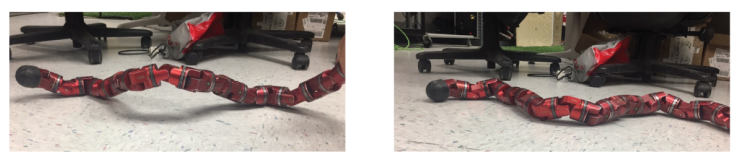

Fig. 2. Examples of Statically Stable and Unstable Configurations (a) The contact state pattern and an example of statically stable configuration for gaits with high spatial frequency in both the lateral wave and the vertical wave. (b.1) The contact state pattern and an example of the statically unstable configuration for gaits with low spatial frequency in both the lateral wave and the vertical wave. (b.2) Stabilizing the statically unstable configuration by increasing the vertical spatial frequency. The label and the axis in panel (b) are the same as in (a). (c) Example of an unstable configuration (left) and an unexpected touchdown (right)

lateral and vertical planes are required.

In this paper, we apply geometric mechanics tools [5, 11, [19, 3] to coordinate motions in the lateral plane and the contact pattern regulation and therefore produce motions in a desired direction. We use our approach to stabilize the statically unstable sidewinding gaits. As a result, we obtain effective and statically stable sidewinding gaits in the desired directions (lateral or rotational). We validate our theoretical predictions through a series of experiments with a 15-DoF Dynamixel motor limbless robot (Fig. 1b.1) and a 16-DoF serial elastic actuated (SEA) limbless robot (Fig. 1b.2) locomoting on flat hard ground.

\section{RELATED WORK}

\section{A. Sidewinder Locomotion}

Sidewinding locomotion has been described as the superposition of two traveling waves: one in the lateral direction and another in the vertical direction [1, 12]. To allow for motion in 3D environments for limbless robots, the motors that form robots were connected such that the orientation of the plane of actuation was offset by $90^{\circ}$ with respect to adjacent modules. Each module has one rotational degree of freedom, with angular orientation ranging from $-90^{\circ}$ to $90^{\circ}$. For an $N$ DoF limbless robot, the links are labeled from 0 to $N$; joints are labeled 1 to $N$, where joint $i$ immediately precedes link $i$ and therefore moves link $i$ and all other links distal to joint $i$. Odd numbered joints are lateral and therefore produce motion in the horizontal plane (and their axes are vertical). Even numbered joints are vertical and therefore produce motion in the vertical plane (and their axes of rotation are horizontal). The joint angles are described using the following functions:

$$
\begin{gathered}
\theta(2 i-1, t)=A_{l} \sin \left(2 \pi K_{l} \frac{2 i-1}{N}+2 \pi f t\right), \\
\theta(2 i, t)=A_{v} \sin \left(2 \pi K_{v} \frac{2 i}{N}+2 \pi f t+\phi_{0}\right),
\end{gathered}
$$

where $\theta_{l}(2 i-1, t)$ and $\theta_{v}(2 i, t)$ refer to the lateral (odd)joint angles and the (even) vertical joint angles respectively; $K_{l}$ and $K_{v}$ are the spatial frequency of the lateral wave and the vertical wave respectively; $A_{l}$ and $A_{v}$ are the amplitude of the lateral wave and the vertical wave respectively; $f$ defines the temporal frequency; and $\phi_{0}$ is the phase lag between the lateral and the vertical wave.

The contact state of link $i$ is represented by $c(i)$, where $c(i)=1$ indicates that link $i$ is on contact and $c(i)=0$ indicates that link $i$ is not in contact. The links between two consecutive vertical joints have the same contact state, i.e., $c(2 i)=c(2 i-1)$. Therefore, the contact state can be approximated by [16]:

$$
c(2 i-1, t)=c(2 i, t)=\sigma\left(\sin \left(2 \pi K_{v} \frac{i}{2 N}+2 \pi f t+\phi_{0}\right)\right),
$$

where $\sigma(x)=\frac{1}{1+e^{-\gamma x}}$, with the parameter $\gamma$ modulating the smoothness of the contact state transition. In this paper, we take $\gamma=4$ unless otherwise stated.

Previous work [12] demonstrated that during sidewinding locomotion, where the locomotors have lateral translational displacement with no significant turning, $K_{l}=K_{v}$ and $\phi_{0}=\pi / 2$. Manipulation of the vertical amplitude $A_{v}$ can change the body contact ratio (average percentage of the body that is in contact with the environment), and therefore enables climbing on sandy slopes. Hu et al. [8] showed that snakes lifted the body portion with the largest curvature during slithering locomotion, so this locomotion has $K_{v}=2 K_{l}$ and $\phi_{0}=0$. In addition to the translational motions, modulating the ratio of the spatial frequency in the vertical and lateral directions, $K_{v} / K_{l}$, can yield turning gaits [1]. Either increasing $\left(K_{v}=1.3 K_{l}\right)$ or decreasing $\left(K_{v}=0.6 K_{l}\right)$ the vertical spatial frequency will both lead to clockwise turning. We summarize previous work on wave modulation in Table 1 .

\section{B. Geometric mechanics}

In this subsection, we provide a concise overview of the geometric tools, which we use to design the coordination between 


\begin{tabular}{|c|c|c|c|c|}
\hline$K_{v}$ & $K_{v} / K_{l}$ & $\phi_{0}$ & Behavior & Source \\
\hline 1.5 & 1 & $\pi / 2$ & Sidewinding & {$[12]$} \\
\hline 2 & 1 & $\pi / 2$ & Sidewinding & {$[1]$} \\
\hline 1.5 & 0.6 & $\pi / 2$ & Clockwise turning & {$[1]$} \\
\hline 1.5 & 1.3 & $\pi / 2$ & Clockwise turning & {$[1]$} \\
\hline 1.5 & 2 & 0 & Slithering & {$[8]$} \\
\hline
\end{tabular}

TABLE I

SUMMARY OF PREVIOUS WORK ON SIDEWINDER LOCOMOTION.

the lateral wave and the vertical wave. For a more detailed and comprehensive review, we refer readers to [5, 11, 19, 3]. The geometric mechanics gait design framework separates the configuration space of a system into two spaces: a position space and a shape space. The position space represents the location (position and rotation) of a system relative to the world frame, while the shape space denotes the internal shape of the system. The geometric mechanics framework then establishes a functional relationship to map the velocities in the shape space to the velocities in the position space; this functional relationship is often called a connection.

1) Kinematic reconstruction equation: In kinematic systems where inertial effects are negligible, the equations of motion [11] reduce to

$$
\xi=A(r) \dot{r},
$$

where $\boldsymbol{\xi}=\left[\begin{array}{lll}\xi_{x} & \xi_{y} & \xi_{\theta}\end{array}\right]^{T} \in g$ denotes the body velocity in the forward, lateral, and rotational directions; $r$ denotes the internal shape variables (joint angles); $\boldsymbol{A}(\boldsymbol{r})$ is the local connection matrix, which encodes environmental constraints and the conservation of momentum. As shown in [7], the local connection matrix, $\boldsymbol{A}$, can be numerically derived by force and torque balance. It is challenging to model the friction precisely, especially when there is a variety of contact surfaces. However, as shown in [16], modeling movement on hard ground using a kinetic Coulomb friction force model has produced good agreement with experimental data.

2) Connection vector fields and height functions: Each row of the local connection matrix, $\boldsymbol{A}$, corresponds to a component direction of the body velocity. Each row of the local connection matrix over the shape space then formed a connection vector field. In this way, the body velocities in the forward, lateral, and rotational directions are respectively computed as the dot product of connection vector fields and the shape velocity $\dot{\boldsymbol{r}}$.

A periodic gait can be represented as a closed curve in the corresponding shape space. The displacement resulting from a gait, $\partial \chi$, can be approximated by:

$$
\left(\begin{array}{c}
\Delta x \\
\Delta y \\
\Delta \theta
\end{array}\right)=\int_{\partial \chi} A(r) d r .
$$
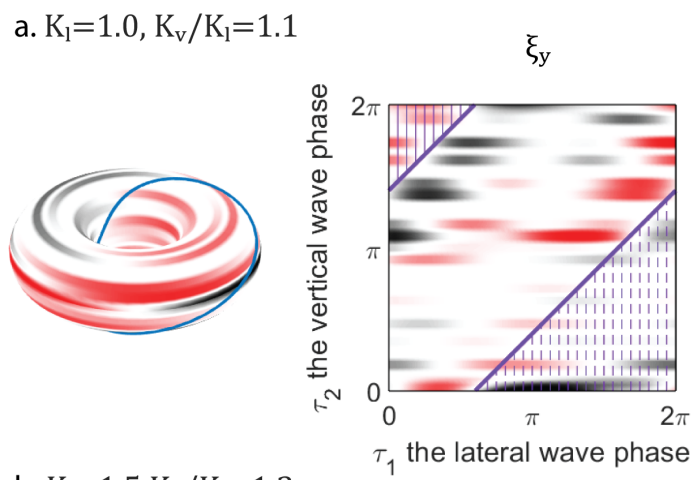

b. $\mathrm{K}_{\mathrm{l}}=1.5 \mathrm{~K}_{\mathrm{v}} / \mathrm{K}_{\mathrm{l}}=1.3$

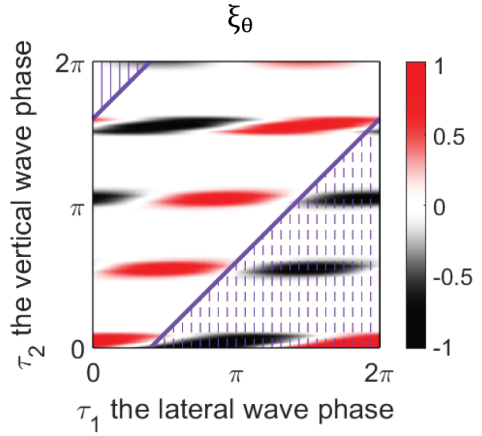

Fig. 3. Height functions to design gaits to produce motion in the desired direction. The height function in the lateral (b) and rotational (a) height functions for (a) lateral spatial frequency $K_{l}=1.5$, V-L ratio $K_{v} / K_{l}=1.3$ and (b) lateral spatial frequency $K_{l}=1.0, \mathrm{~V}-\mathrm{L}$ ratio $K_{v} / K_{l}=1.2$. The purple curve in each plot maximizes the surface integral enclosed in the upper left corner (marked in solid lines) minus the surface integral enclosed in the lower left corner (marked in the dashed lines). The units and the axis labels in all panels are the same.

According to Stokes' Theorem, the line integral along a closed curve $\partial \chi$ is equal to the surface integral of the curl of $\boldsymbol{A}(\boldsymbol{r})$ over the surface enclosed by $\partial \chi$ :

$$
\int_{\partial \chi} \boldsymbol{A}(\boldsymbol{r}) \boldsymbol{d} \boldsymbol{r}=\iint_{\chi} \nabla \times \boldsymbol{A}(\boldsymbol{r}) \boldsymbol{d} r_{1} d r_{2},
$$

where $\chi$ denotes the surface enclosed by $\partial \chi$. The curl of the connection vector field, $\boldsymbol{\nabla} \times \boldsymbol{A}(\boldsymbol{r})$, is referred to as the height function. The three rows of the vector field $A(r)$ can thus produce three height functions in the forward, lateral and rotational direction, respectively.

With the above derivation, we simplify the gait design problem to drawing a closed path in a Euclidean shape space. Displacements can be approximated by integral of the surface enclosed by the gait path.

3) Torus shape space: In the sidewinder gait prescription, with both shape variables being cyclic, the shape space is toroidal, $\left(T^{2}\right)$ [10]. Some examples of height functions on toroidal shape spaces are shown in Fig. 3 The shape variable, $\boldsymbol{\tau}=\left[\tau_{1}, \tau_{2}\right]^{T} \in T^{2}$, has a cyclic structure, where one axis represents the phase of the lateral wave, and the other axis represents the phase of the vertical wave. While the gait path (solid purple curve Fig. 3 is a closed curve in the toroidal shape space, there is no obvious surface enclosed by the gait path. 
a. $\mathrm{K}_{\mathrm{l}}=1.0, \mathrm{~K}_{\mathrm{v}} / \mathrm{K}_{\mathrm{l}}=1.0$

b. $\mathrm{K}_{\mathrm{l}}=1.5, \mathrm{~K}_{\mathrm{v}} / \mathrm{K}_{\mathrm{l}}=1.0$
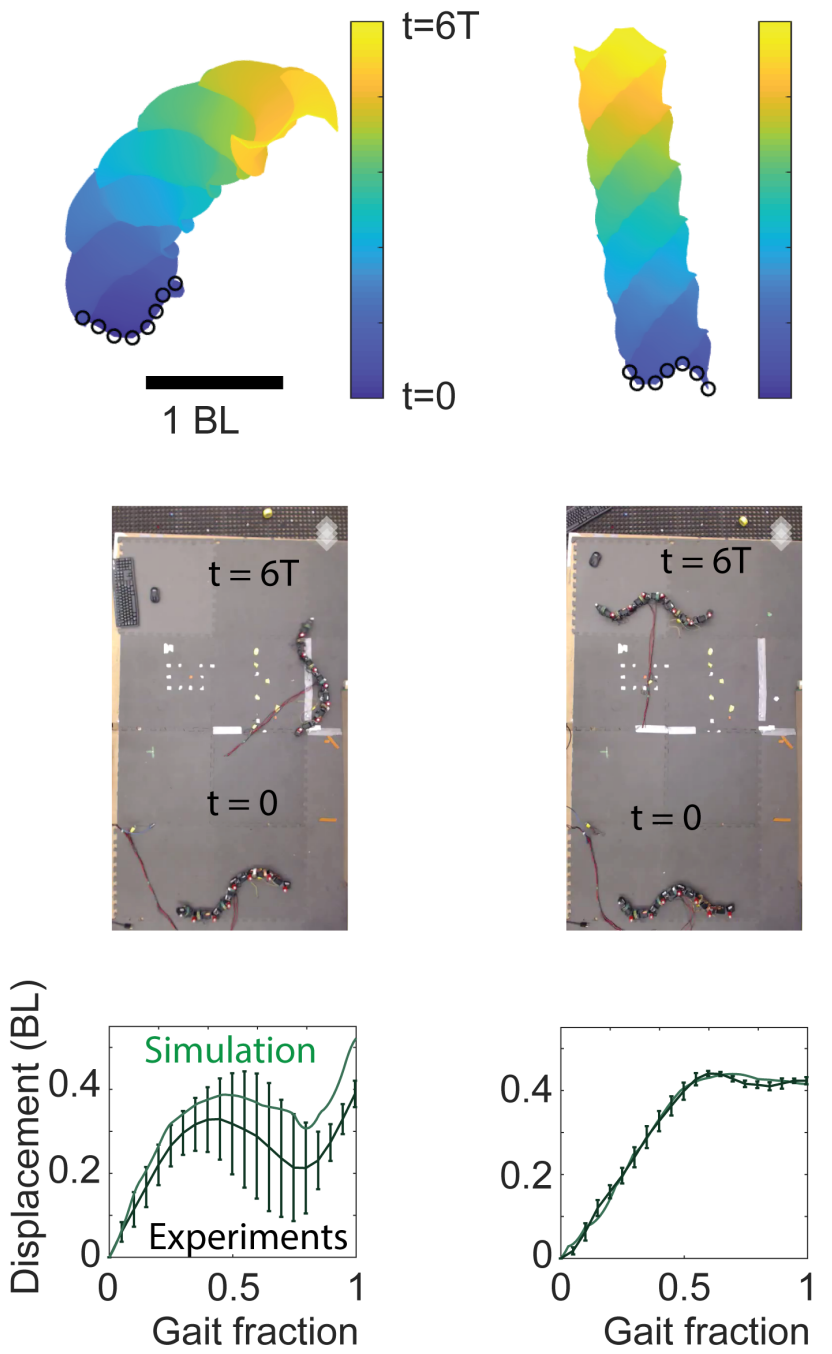

Fig. 4. Discrepancy between Dynamixel robot experiments and simulation at low spatial frequency. (top) The trajectories of body motion in 6 gait cycles. The colors represent gait periods. Initial positions of the Dynamixel robot indicated by the black circles. (Bottom) Comparisons of time evolution of displacement of the simulation and Dynamixel robot experiments. We compared the low spatial frequency gait (a) and high spatial frequency gait (b). The simulation-experiment discrepancy occurs in low spatial frequency gaits. The unit and the axis labels in all panels are the same.

To form an enclosed surface, Gong et al. [5] introduced the notion of two "assistive lines" in the Euclidean parameterization of the toroidal shape space. As a result, the surface integral can be calculated as the surface enclosed in the upper left corner (see the surface labeled by solid lines in Fig. 3) minus the surface enclosed in the lower right corner (see the surface labeled by dashed lines in Fig. 3. Finally, we refer readers to [5, 3] for a detailed derivation and proof of motion planning in toroidal shape spaces.
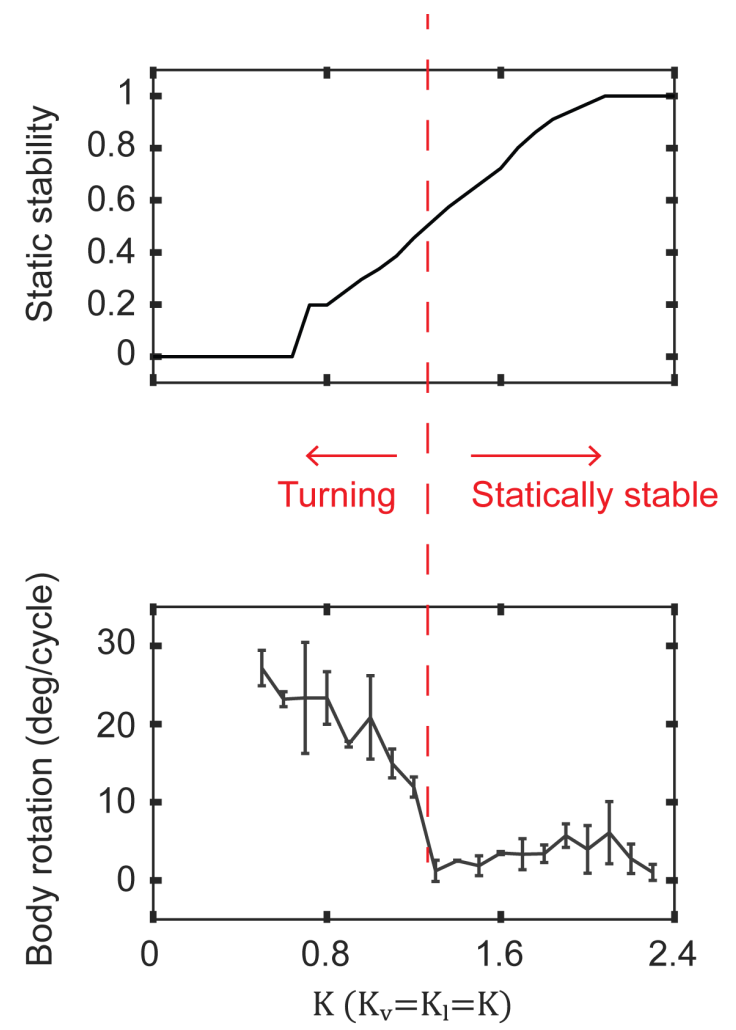

Fig. 5. Effect of spatial frequency on the static stability. The figure on top panel shows the relationship between the spatial frequency $\left(K_{v}=K_{l}=\right.$ $K$ ) and the static stability (top panel). Dynamixel robot experiments showed significant turning were observed in gaits with low static stability and the turning vanished at gaits with high static stability (bottom panel).

\section{iII. Frequency Modulation to Stabilize Gaits}

\section{A. Sidewinder Gait Formula}

1) Vertical Wave Prescription: As mentioned in Sec. III the contact pattern can be approximated by Eq. 3 for the traveling wave (Eq. 2) in the vertical joint angles. In Fig. 2, we showed the example of the contact sequence for $K_{v}=1.5,1.0$ and 1.1. To produce the contact pattern that can be precisely described in Eq. 3, we replace the vertical joint angle formula by:

$$
\theta(2 i, t)=A_{v}(-c(2 i, t)+c(2 i+1, t)) .
$$

Note that in our experiments on the SEA robot, joint $N$ (the last joint) always orients link $N$ (the tail link) into the air so the tether does not interfere with its motion.

2) Static Stability: Static stability is defined as the fraction of a period that the center of mass is inside the support polygon. The support polygon is defined as the convex hull of all the links in contact with substrate. In Fig. 2, we show the example of stable configurations (Fig. 2a) and unstable configuration (Fig. $2 \mathrm{p}$ ). We observe that gaits with high vertical spatial frequencies have more distinct body-environment contact patches, and are therefore more statically stable than 
a. $\mathrm{K}_{\mathrm{l}}=1.0, \mathrm{~K}_{\mathrm{v}} / \mathrm{K}_{\mathrm{l}}=1.0$

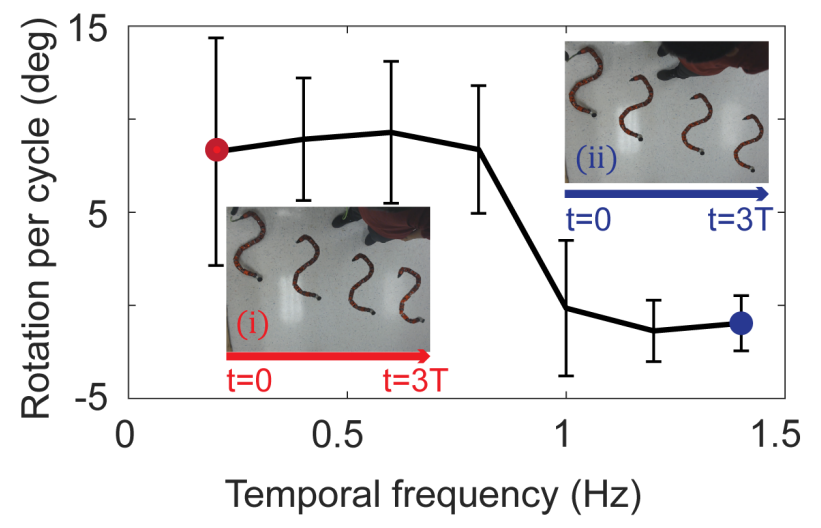

b. $\mathrm{K}_{\mathrm{l}}=1.5, \mathrm{~K}_{\mathrm{v}} / \mathrm{K}_{\mathrm{l}}=0.6$

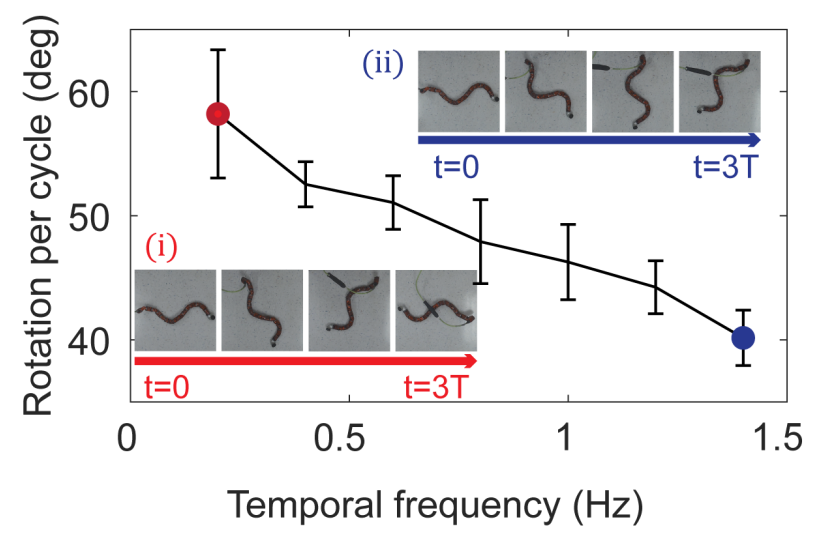

Fig. 6. Temporal frequency dependency of unstable gaits. Dependence of the rotation angle (per cycle) on the temporal frequency of (a) statically unstable sidewinding gaits and (b) statically unstable turning gaits on SEA robot experiments. The subplots (i) and (ii) show the snapshots of the SEA robot implementing gaits in low temporal frequency $(0.2 \mathrm{~Hz}$, red) and high temporal frequency $(1.4 \mathrm{~Hz}$, blue) over three gait cycles.

gaits with low spatial frequency in the vertical wave. We will use this observation to stabilize the original unstable gaits.

3) Gait Stabilization: We stabilize the sidewinding and turning gait by increasing the spatial frequency of the vertical wave. In other words, we gradually increase the V-L ratio, $K_{v} / K_{l}$, until the satisfactory static stability is reached. In this paper, we consider a gait as statically stable when its static stability is greater than 0.5 . Note that this threshold is manually selected for our experiments on flat terrain. We can raise the static stability threshold in the applications where the robots need to traverse rough terrain.

4) Coordination of the Lateral and the Vertical Wave: We decomposed the motion of sidewinder gaits as two independent traveling waves: the lateral traveling wave and the vertical traveling wave. The lateral traveling wave is prescribed by:

$$
\theta_{l}\left(j, \tau_{1}\right)=A_{l} \sin \left(2 \pi K_{l} \frac{j}{N}+\tau_{1}\right),
$$

where $\tau_{1}$ is the phase of the lateral wave. Similarly, the contact a. $\mathrm{K}_{\mathrm{l}}=1.0, \mathrm{~K}_{\mathrm{v}} / \mathrm{K}_{\mathrm{l}}=1.1$

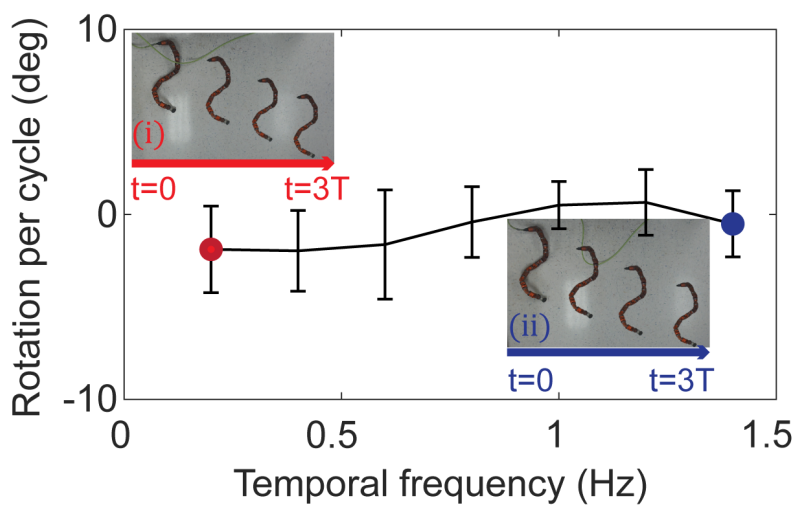

b. $\mathrm{K}_{\mathrm{l}}=1.5, \mathrm{~K}_{\mathrm{v}} / \mathrm{K}_{\mathrm{l}}=1.3$

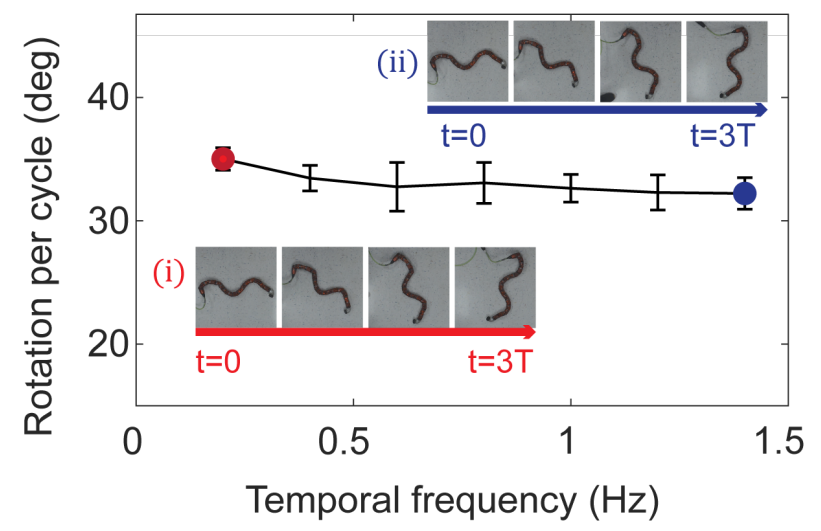

Fig. 7. Robustness of statically stable gaits over temporal frequency. Dependence of the rotation angle (per cycle) on the temporal frequency of (a) the stabilized sidewinding gait and (b) the stabilized turning gait on SEA robot experiments. In both cases, the rotation angle is steady over a range of temporal frequencies. The unit and the axis labels in all panels are the same. The subplots (i) and (ii) show the snapshots of the SEA robot implementing gaits in low temporal frequency $(0.2 \mathrm{~Hz}$, red) and high temporal frequency $(1.4 \mathrm{~Hz}$, blue) over three gait cycles.

state is prescribed as:

$$
c\left(2 i-1, \tau_{2}\right)=c\left(2 i, \tau_{2}\right)=\sigma\left(\sin \left(2 \pi K_{v} \frac{i}{2 N}+\tau_{2}\right)\right)
$$

where $\tau_{2}$ is the phase of the vertical wave that can uniquely determine the contact pattern. $c\left(i, \tau_{2}\right)=0$ represents that no ground reaction force appears at link $i$ at phase $\tau_{2}$.

The phases of the lateral wave and the vertical wave then comprise the shape variable, $\boldsymbol{\tau}=\left[\begin{array}{ll}\tau_{1}, & \tau_{2}\end{array}\right]^{T}$. Using the geometric mechanics gait design tools mentioned in Sec. II we we can calculate the height function and visualize the kinematics in the desired directions (lateral and rotational).

A gait that coordinates the lateral and vertical wave can be described as $\left\{\Phi: \tau_{1} \longrightarrow \tau_{2}\right\}$. From the pattern of the height functions (see Fig. 3), we observed that in the parameterization of the torus (where the edges are properly identified with each other at 0 and $2 \pi$ ), a straight line path gives rise to a optimal path; this is seen by the integral of the surface in the upper left minus the integral of the surface in the lower right being 
maximized. In this way, we characterize the coordination of the lateral and the vertical wave by the relative phase lag: $\phi_{0}:=\left(\tau_{2}-\tau_{1} \bmod 2 \pi\right)$

\section{B. Numerical Simulation}

We performed the numerical simulation to predict the locomotion performance and compared it to the robots experiments. Specifically, in the simulation, we prescribed the lateral amplitude and the contact state of the robot using Eq. 1 and Eq. 3. In other words, we take $\tau_{1}=2 \pi f t$ and $\tau_{2}=2 \pi f+\phi_{0}$. Thus, the shape variable and shape velocity can be prescribed as:

$$
\boldsymbol{\tau}=\left[\begin{array}{c}
2 \pi f t \\
2 \pi f t+\phi_{0}
\end{array}\right], \dot{\boldsymbol{\tau}}=\left[\begin{array}{c}
2 \pi f \\
2 \pi f
\end{array}\right]
$$

Then we simulate the locomotion with the standard ordinary differential equation [6]:

$$
\begin{aligned}
g(T) & =\int_{0}^{T} T_{e} L_{g(t)} A(\boldsymbol{\tau}) \dot{\boldsymbol{\tau}} \boldsymbol{d} t \\
& =\int_{0}^{T} T_{e} L_{g(t)} A\left(\left[\begin{array}{c}
2 \pi f t \\
2 \pi f t+\phi_{0}
\end{array}\right]\right)\left[\begin{array}{c}
2 \pi f \\
2 \pi f
\end{array}\right] \boldsymbol{d} t,
\end{aligned}
$$

where $g=(x, y, \alpha) \in S E(2)$ represents the body frame

position and rotation [15], $T_{e} L_{g}=\left[\begin{array}{ccc}\cos (\alpha) & -\sin (\alpha) & 0 \\ \sin (\alpha) & \cos (\alpha) & 0 \\ 0 & 0 & 1\end{array}\right]$ is the left lifted action with respect to the coordinates of $g$. Kinetic Coulomb friction friction were used to derive the local connection matrix, $A$.

Solving the ordinary differential equation throughout one period (from $t=0$ to $t=1 / f$ ), we obtain the trajectory of locomotor and can determine the predicted displacements in the forward, lateral, and rotational directions over one gait cycle. Note that we neglect the inertia effect in the simulation.

Note that we assume that the friction force dominates the motion and we neglect the inertia in the simulation. However, the inertia can be important in the gait stability. That is, when the statically unstable gaits are implemented on robots with low temporal frequency (i.e., no gained dynamic stability), the robot often cannot reach the prescribed configuration; therefore, simulation-experiment discrepancy is expected (see Fig. 3a). On the other hand, when operated at high temporal frequency, inertia effects can make the statically unstable gaits dynamically stable. In the case where the gait can be stably (either statically stable or dynamically stable) implemented on robots, the inertia has a relatively small contribution to the motion (i.e., friction dominates the motion), as we showed in Fig. $3 b$.

\section{SEA robot and Dynamixel robot experiments}

We carried out experiments with a Dynamixel robot (Fig. 1p.1) and a serial elastic actuated (SEA) robot (Fig. 1p.2). The Dynamixel robot is a modular snake robot composed of 15 identical actuated modules (Dynamixel AX-12A servo motors) connected with 3D printed plastic brackets; the SEA robot is a modular series elastic actuated robot composed of a chain of 16 identical modules that are capable of precise torque, velocity and position control [17]. The arrangement of modules in the SEA robot and the Dynamixel robot makes sure that the rotation axes of neighbouring modules were $90^{\circ}$ rotated in a torsional manner. Note that during the experiments, the connection wire is lifted to avoid the additional force by the connection wire. Thus, the joints were divided into two groups: yaw joints (odd modules from head to tail), which control the lateral body wave, and pitch joints (even modules), which control the vertical body wave.

Experiments were conducted on the flat hard ground, where we assume the ground reaction forces are given by kinetic Coulomb friction. The SEA robot and the Dynamixel robot were controlled directly by joint angle commands. For each sidewinder gait tested, we conducted 5 trials. In each trial, we commanded the SEA robot to execute three complete gait cycles and collected the SEA robot motion data starting from the first command being sent out until the SEA robot stop moving.

To track the motion of the SEA robot in the environment, we uniformly attached 8 IR reflective markers along the body of the SEA robot and the Dynamixel robot. An OptiTrack motion capture system was employed, and 4 OptiTrack Flex 13 cameras were installed to track the three-dimensional positions of the markers at 120 FPS frame rate. We recorded the trajectory of the markers over 3 cycles, from which we calculate the forward, lateral and rotational displacements.

The Dynamixel robot and the SEA robot are used in different experiments. Specifically, we used the Dynamixel robot to test the effects of spatial frequency on static stability. In these experiments, different gaits are implemented on the robot at the same temporal frequency. Note that the maximum allowable torque of the Dynamixel motors is $1.5 \mathrm{~N}-\mathrm{m}$. To avoid large torques on the motors, we ran experiments on the Dynamixel robot at relatively low temporal frequency. In our efforts to stabilize gaits, we measured the stability of a gait by testing it over different temporal frequency. In this case, we unavoidably need to run experiments in relatively high temporal frequency and therefore large torque is expected on motors. The insufficient torque can restrict the capacity of the robot to form the desired shape. In this way, we used the SEA robot, which has the maximum allowable torque $7 \mathrm{~N}$-m, to test the effect of temporal frequency on static stability. Examples of the SEA robot and the Dynamixel robot experiments can be found in the supplementary video.

We summarize our steps to stabilize the sidewinding gaits in Algorithm 1

\section{Results}

\section{A. Effect of Spatial Frequency on Static Stability}

Previous work [16] suggested that for sidewinding gaits with 1.5 spatial waves $\left(K_{l}=1.5, K_{v} / K_{l}=1.0\right)$ are in general faster than the sidewinding gaits with 2.0 spatial waves $\left(K_{l}=\right.$ 


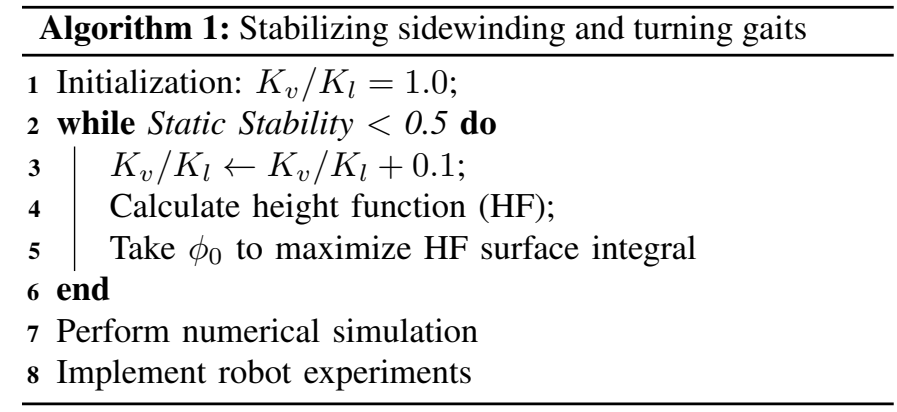

2.0, $\left.K_{v} / K_{l}=1.0\right)$. In both cases, the gaits are statically stable and the simulation can well predict the trajectory of body motion and have good agreement with Dynamixel robot experiments. With this knowledge, we proceed to study the locomotion performance of the statically unstable sidewinding gaits.

We calculated the static stability for sidewinding gaits with different spatial frequencies $\left(K_{v}=K_{l}=K\right)$ in Fig. 5 . High spatial frequencies will lead to dense distribution of short contact patch (Fig. 2a) and often is statically stable. On the other hand, low spatial frequencies will lead to sparse distribution of long contact patch (Fig. 2p.1) and is often not statically stable.

To investigate the behavior of statically unstable sidewinding gaits, we performed the similar experiments on sidewinding gaits with 1.0 spatial wave and 1.5 waves on our Dynamixel robot (Fig. 1p.1). We take lateral amplitude $A_{l}=$ $0.15 K_{v}$ rad and vertical amplitude $A_{v}=0.7 K_{v}$ rad, temporal frequency $f=1 \mathrm{~Hz}$ for all the Dynamixel robot experiments. Snapshots of Dynamixel robots implementing gaits are shown in Fig. 4. Good agreement between experiment and theory was observed in sidewinding gait with 1.5 spatial waves. However, we observed significant discrepancies between the simulation and Dynamixel robot experiments (see Fig. 4). We hypothesized that at low spatial frequency, the configuration of the robot would not be statically stable (static stability = 0.48 for 1.0 spatial wave, static stability $=0.78$ for 1.5 spatial waves) and this led to the falling down of robot (see Fig. $2 \mathrm{p}, \mathrm{c})$. The unexpected touchdown can change the distribution of ground reaction forces and therefore leads to turning.

To verify our hypothesis, we ran Dynamixel robot experiments across a range of spatial frequencies. Dynamixel robot experiments showed that such discrepancies (stability related turning) vanished at high spatial frequencies. From the Dynamixel robot experiment result, we observed that, in this experiment set-up, the cut-off static stability that leads to unexpected behavior is around 0.5 .

\section{B. Temporal Frequency Dependency}

Although some gaits are not statically stable, it is possible that increasing the temporal frequency can make gaits dynamically stable. Thus, we tested the effect of the temporal frequency on the performance of gaits.

We first evaluate the effect of temporal frequency on the sidewinding gait with 1.0 spatial wave $\left(K_{l}=1.0, K_{v} / K_{l}=\right.$ 1.0). We take $A_{v}=75^{\circ}$ and $A_{v}=50^{\circ}$ for all the SEA robot experiments. At low temporal frequency (see Fig. 7), rotations with high mean and high standard deviation were observed in SEA robot experiments, which suggested that the locomotion performance for static unstable gaits was not predictable and controllable when operating at different speeds. However, both the mean and the deviation of the rotation significantly decreased when the SEA robot operates at high temporal frequency. It suggested that the loss of static stability can be compensated by the obtained dynamic stability at high speed.

Next, we evaluate the temporal frequency dependence of the frequency turning gait from [1]: $K_{l}=1.5, K_{v} / K_{l}=0.6$, $\phi_{0}=\pi / 2$. From our static stability analysis, the frequency turning gait is not statically stable (static stability $=0.46$ ). In addition, numerical simulation suggested that the frequency turning gaits should lead to counterclockwise rotation, in contradiction with the experiments. Therefore we speculate that the frequency turning gait is driven by the unexpected touchdowns and therefore will be strongly temporal frequency dependent. SEA robot experiments verified that locomotion performance in the frequency turning gait is strongly correlated with the temporal frequency. Higher rotation angles were achieved when the SEA robot operated at low temporal frequency.

\section{Stabilization by Modulation of the Vertical Waves}

We followed the algorithms proposed in section III to stabilize the statically unstable sidewinding and turning gait. As discussed earlier, the sidewinding gait with 1.0 spatial wave is not statically stable. We showed that we can stabilize this gait by increasing the V-L ratio $K_{v} / K_{l}$ to 1.1 . From the lateral height function (Fig. 3p), we take $\phi_{0}=1.29$ to optimize the surface enclosed in the lateral height function. The static stability analysis suggested that this gait is statically stable ( static stability $=0.62$ ). We implemented this gait on SEA robot experiments. SEA robot experiments (Fig. 7a) showed that no significant turning was observed over a range of temporal frequencies.

Note that the stabilized sidewinding gait $\left(K_{l}=1.0\right.$, $\left.K_{v} / K_{l}=1.1\right)$ exhibited excellent lateral speed. SEA robot experiments shows that the average lateral displacement per gait cycle is $0.45 \pm 0.03$ body length, significantly greater than the displacement $(0.37 \pm 0.01$ body length per gait cycle) of sidewinding gait with 1.5 spatial waves $\left(K_{l}=1.5\right.$, $\left.K_{v} / K_{l}=1.0\right)$.

We next stabilized the frequency turning gait with 1.5 spatial waves, $K_{l}=1.5$. We showed that we can stabilize it by raising the $\mathrm{V}-\mathrm{L}$ ratio $K_{v} / K_{l}$ to 1.3 . From the rotational height function (Fig. 33), we take $\phi_{0}=1.02$ to optimize the surface enclosed in the lateral height function. The static stability analysis suggested that this gait is statically stable ( static stability =0.62). We implemented this gait on the SEA robot. SEA robot experiments showed that the locomotion performance (rotation per gait cycle) is robust over a range of temporal frequencies. 


\section{DISCUSSION}

\section{A. Sidewinding Gait Family}

In previous work [1, 12], sidewinding gaits have been described as the superposition of the body wave in the lateral and the vertical directions of the same spatial frequency $\left(K_{v}=K_{l}\right)$. This equality limits the availability of sidewinding gaits, especially at low temporal frequency. In this work, we have shown that $K_{v}=K_{l}$ is not necessary to produce sidewinding locomotion. In fact, for almost any V-L ratio, $K_{v} / K_{l}$, we can always find a $\phi_{0}$ to produce sidewinding. This result greatly expands the effective sidewinding gait family operating at a wide spectrum of temporal frequencies.

In our expanded sidewinding gait family, greater V-L ratio in general corresponds to faster but less stable locomotion; less V-L ratio in general corresponds to slower but more stable gaits. By modulating the V-L ratio, we can systematically do the trade-off between the speed and stability.

\section{B. Mechanisms of Frequency Turning Gaits}

Turning motions of limbless robots have been less studied than translational motion. Frequency turning (modulating the $\mathrm{V}-\mathrm{L}$ ratio with fixed phase lag $\phi_{0}=\pi / 2$ ) is one of the effective in-place turning strategies for limbless locomotors [1]. However, the mechanism of frequency turning gaits was not identified.

In this paper, we studied frequency turning gaits. Static stability analysis shows that the frequency turning gaits with V-L ratio greater than 1 are statically stable. Moreover, SEA robot experiments showed that the locomotion performance of frequency turning gaits with $\mathrm{V}-\mathrm{H}$ ratio greater than 1 is not temporal frequency dependent. Geometric height functions indicate that it is the changes in the symmetry of ground reaction forces that lead to the changes in the direction of motion.

However, in the simulation and geometric mechanics prediction, the frequency turning gaits should lead to counterclockwise turning when the V-L ratio is less than 1 , which contradicts the experimental results. Furthermore, static stability analysis shows that the frequency turning gaits with V-L ratio less than 1 are not statically stable. SEA robot experiments also suggested that their locomotion performances are not robust over the temporal frequencies. In this way, we speculate that the frequency turning gaits with V-L ratio less than 1 are driven by the additional ground reaction forces induced by static instability.

\section{CONCLUSION}

This paper provided the first observations of the impact of temporal frequency on the locomotion performance of limbless locomotors. As with previous work, we prescribed the motion of limbless locomotors by two waves: one wave in the vertical plane and the other in the horizontal. We show that, at low temporal frequency, statically unstable sidewinding gaits have undesirable locomotion performance and deviated from model expectations. In other words, some gaits were effective only at high temporal frequency, where the static instability can be compensated by the gained dynamic stability. In fact, the loss in static stability limits the feasible choices of sidewinding gaits at low temporal frequency.

We proposed an approach to stabilize these statically unstable sidewinding gaits by modulating the spatial frequency of the vertical wave. We used height functions, analytic tools previously described from the geometric mechanics literature, to coordinate the lateral wave and vertical wave to produce effective motion in the desired directions. Robot experiments verified that the temporal frequency dependence were eliminated in statically stable turning gaits.

In this way, we greatly expanded the range of statically stable sidewinding gaits by introducing another control variable $K_{v} / K_{l}$ to trade-off between the static stability and the speed. Robots in real world need not only effective performance at high speed, but also stable operation at low speed. For example, in the control of load-bearing robots and navigation through cluttered search and rescue environments, stability is often more important than speed. In our proposed framework, we observed a lower bound of the temporal frequencies where the dynamic stability fails to compensate for the loss of static stability. Therefore, it is important to stabilize the gaits to allow effective locomotion performance at low temporal frequencies.

In the future, we will study the gait optimization with the combined effect of static stability and dynamic stability and further improve the gait adaptations in response to the changes in speed. In addition, we will expand our model to advance hypotheses for further study of biological sidewinder snakes at varying speeds. 


\section{REFERENCES}

[1] Henry C Astley, Chaohui Gong, Jin Dai, Matthew Travers, Miguel M Serrano, Patricio A Vela, Howie Choset, Joseph R Mendelson, David L Hu, and Daniel I Goldman. Modulation of orthogonal body waves enables high maneuverability in sidewinding locomotion Proceedings of the National Academy of Sciences, 112(19): 6200-6205, 2015.

[2] Yasemin Ozkan Aydin, Jennifer M Rieser, Christian M Hubicki, William Savoie, and Daniel I Goldman. Physics approaches to natural locomotion: Every robot is an experiment In Robotic Systems and Autonomous Platforms, pages 109-127. Elsevier, 2019.

[3] Baxi Chong, Yasemin Ozkan Aydin, Guillaume Sartoretti, Jennifer M Rieser, Chaohui Gong, Haosen Xing, Howie Choset, and Daniel I Goldman. A Hierarchical Geometric Framework to Design Locomotive Gaits for Highly Articulated Robots In Robotics: science and systems, 2019.

[4] Chaohui Gong, Matthew Travers, Henry C Astley, Daniel I Goldman, and Howie Choset. Limbless locomotors that turn in place. In 2015 IEEE International Conference on Robotics and Automation (ICRA), pages 3747-3754. IEEE, 2015.

[5] Chaohui Gong, Julian Whitman, Jaskaran Grover, Baxi Chong, Richard Ren, and Howie Choset. Geometric motion planning for systems with toroidal and cylindrical shape spaces In Dynamic Systems and Control Conference, 2018.

[6] Ross L Hatton and Howie Choset. Nonconservativity and noncommutativity in locomotion. The European Physical Journal Special Topics, 224(17-18):3141-3174, 2015.

[7] Ross L Hatton, Yang Ding, Howie Choset, and Daniel I Goldman. Geometric visualization of self-propulsion in a complex medium Physical review letters, 110(7): 078101, 2013.

[8] David L Hu, Jasmine Nirody, Terri Scott, and Michael J Shelley. The mechanics of slithering locomotion Proceedings of the National Academy of Sciences, 106(25): 10081-10085, 2009.

[9] Bruce C Jayne. Kinematics of terrestrial snake locomotion Copeia, pages 915-927, 1986.

[10] Shoshichi Kobayashi and Katsumi Nomizu. Foundations of differential geometry, volume 1. Interscience publishers New York, 1963.

[11] Jerrold E Marsden and Tudor S Ratiu. Introduction to mechanics and symmetry: a basic exposition of classical mechanical systems volume 17. Springer Science \& Business Media, 2013.

[12] Hamidreza Marvi, Chaohui Gong, Nick Gravish, Henry Astley, Matthew Travers, Ross L Hatton, Joseph R Mendelson, Howie Choset, David L Hu, and Daniel I Goldman. Sidewinding with minimal slip: Snake and robot ascent of sandy slopes. Science, 346(6206):224229, 2014.
[13] Tad McGeer et al. Passive dynamic walking I. J. Robotic Res., 9(2):62-82, 1990.

[14] R. B. Mcghee and G. I. Iswandhi. Adaptive Locomotion of a Multilegged Robot over Rough Terrain IEEE Transactions on Systems, Man, and Cybernetics, 9(4): 176-182, April 1979. ISSN 0018-9472. doi: 10.1109/ TSMC.1979.4310180.

[15] Richard M Murray. A mathematical introduction to robotic manipulation. CRC press, 2017.

[16] Jennifer M Rieser, Chaohui Gong, Henry C Astley, Perrin E Schiebel, Ross L Hatton, Howie Choset, and Daniel I Goldman. Geometric phase and dimensionality reduction in locomoting living systems arXiv preprint arXiv:1906.11374, 2019.

[17] David Rollinson, Yigit Bilgen, Ben Brown, Florian Enner, Steven Ford, Curtis Layton, Justine Rembisz, Mike Schwerin, Andrew Willig, Pras Velagapudi, et al. Design and architecture of a series elastic snake robot. In 2014 IEEE/RSJ International Conference on Intelligent Robots and Systems, pages 4630-4636. IEEE, 2014.

[18] Perrin E Schiebel, Jennifer M Rieser, Alex M Hubbard, Lillian Chen, D Zeb Rocklin, and Daniel I Goldman. Mechanical diffraction reveals the role of passive dynamics in a slithering snake. Proceedings of the National Academy of Sciences, 116(11):4798-4803, 2019.

[19] Baxi Zhong, Yasemin Ozkan Aydin, Chaohui Gong, Guillaume Sartoretti, Yunjin Wu, Jennifer Rieser, Haosen Xing, Jeffery Rankin, Krijn Michel, Alfredo Nicieza, et al. Coordination of back bending and leg movements for quadrupedal locomotion. In Robotics: Science and Systems, 2018. 